Article

\title{
Synthesis of 2-Phenylazonaphtho[1,8-ef][1,4]diazepines and 9-(3-Arylhydrazono)pyrrolo[1,2-a]perimidines as Antitumor Agents
}

\author{
Thoraya A. Farghaly ${ }^{1, *}$, Eman M. H. Abbas ${ }^{2}$, Kamal M. Dawood ${ }^{1}$ and Tarek B. A. El-Naggar ${ }^{2,3}$ \\ 1 Department of Chemistry, Faculty of Science, University of Cairo, Giza 12613, Egypt; \\ E-Mail: dr_dawood@yahoo.com \\ 2 Department of Chemistry, Natural and Microbial Products, National Research Center, Dokki, \\ Cairo 12622, Egypt; E-Mails: eman_m69@yahoo.com (E.M.H.A.); \\ telnaggar@yahoo.com (T.B.A.E.-N.) \\ 3 Departamento de Farmacología, Facultad de Farmacia, Universidad Complutense de Madrid, \\ Plaza de Ramón y Cajal s/n, Madrid 28040, Spain \\ * Author to whom correspondence should be addressed; E-Mail: thoraya-f@hotmail.com; \\ Tel.: +202-3567-6608; Fax: +202-3567-6501.
}

Received: 17 November 2013; in revised form: 16 December 2013 / Accepted: 17 December 2013 / Published: 8 January 2014

\begin{abstract}
Two series of naphtho[1,8-ef][1,4]diazepines and pyrrolo[1,2-a]perimidines were prepared starting from 1,8-diaminonaphthalene and hydrazonoyl chlorides. The structures of the products were determined on the basis of their spectral data and elemental analyses. The mechanism of formation of such products was also discussed. The prepared compounds were screened for their antitumor activity against three cell lines, namely, MCF-7, TK-10 and UACC-62, and some derivatives showed promising activity.
\end{abstract}

Keywords: 1,8-diaminonaphthalene; naphtho[1,8-ef][1,4]diazepines; pyrrolo[1,2-a] perimidines; hydrazonoyl chloride; antitumor activity

\section{Introduction}

Naphthalene is important aryl ring found in many biologically active compounds such as antiinflammatory [1], anti-bacterial [2], anti-microbial [3] and anti-cancer agents [4]. 1,8-Diaminonaphthalene was used in synthesis of a limited number of naphtho[1,8-ef][1,4]diazepine derivatives [5-8] and many 
perimidine derivatives [9-12]. Perimidines are an important class of heterocyclic systems due to their biological and pharmaceutical activities $[9,10]$. They have been used as anti-ulcer, anti-microbial, and anti-fungal agents $[11,12]$. Perimidines have also been described as DNA-intercalating and anti-tumor agents against several carcinogenic cell lines [13-16]. Very recently, we reported some perimidine anti-tumor agents that showed good activity against the MCF-7 breast cancer cell line and HEPG-2 liver cancer cell line [17]. In addition, perimidines constitute the core of several dyes [18], and are used in the synthesis of photovoltaic devices [19] and as a source of carbene ligands [20]. Furthermore, pyrrolo[1,2-a] perimidines are an interesting class of fused heterocycles that have wide range of industrial applications as dyes and pigments for plastics [21-24]. From the above findings and in continuation of our research work on the use of hydrazonoyl halides in the synthesis of biologically active heterocycles [25-32], we report here the so far unreported reaction between 1,8-diaminonaphthalene and hydrazonoyl chlorides as a key step for synthesis of the naphtho[1,8-ef][1,4]diazepine derivatives. We also synthesized a new perimidine derivative to study its regioselective reaction towards a number of hydrazonoyl chlorides. The effects of the newly synthesized compounds on the growth of three human cancer cell lines: MCF-7, TK-10, UACC-62, are also examined.

\section{Results and Discussion}

\subsection{Chemistry}

Treatment of 1,8-diaminonaphthalene (1) with the acetylhydrazonoyl chloride 2a in dioxane in the presence of triethylamine at reflux temperature furnished a single product to which the structure 2-(4-methoxyphenylhydrazono)-3-methyl-1H-naphtho[1,8-ef] [1,4] diazepine (3a) was assigned based on the elemental analyses and spectral data of the isolated product. The IR spectrum of the reaction product showed two NH absorption bands at 3,394 and 3,286 $\mathrm{cm}^{-1}$. Moreover, the mass spectrum of the isolated product revealed a molecular ion peak at $\mathrm{m} / \mathrm{z} 330 .{ }^{1} \mathrm{H}-\mathrm{NMR}$ displayed two singlet peaks resonating at 2.25 and 3.58 due to the $\mathrm{CH}_{3}$ and $\mathrm{OCH}_{3}$ protons, respectively, in addition to two broad $\mathrm{D}_{2} \mathrm{O}$-exchangeable signals resonating at 9.98 and 10.10 due to two $\mathrm{NH}$ protons, in addition to an aromatic multiplet in the 6.65-7.92 ppm region.

Analogous reactions of the acetyl hydrazonoyl chlorides $\mathbf{2 b}-\mathbf{j}$ with 1,8-diaminonaphthalene (1) furnished the corresponding 2-(arylhydrazono)-3-methyl-1H-naphtho[1,8-ef][1,4] diazepine derivatives $\mathbf{3 b}-\mathbf{j}$ in high yields, as outlined in Scheme 1. The structures of the isolated products were assigned on the basis of their elemental analyses and spectral data (see Experimental section). Compounds 3 have three tautomeric forms $\mathbf{3 A}-\mathbf{C}$ (Scheme 1). The electronic absorption spectra of $\mathbf{3}$ in dioxane revealed, in each case, one characteristic absorption band in the region 381-345 nm (Table 1) and the electronic absorption spectra of compound 3d, taken in different solvents as a typical example of the series prepared, exhibited little, if any, solvent dependence (Table 1). These data indicate that compound $\mathbf{3}$ is found mainly in the hydrazone-form $\mathbf{3 A}[33,34]$.

Similar reaction of 1,8-diaminonaphthalene (1) with the ester hydrazonoyl chlorides $\mathbf{4 a}, \mathbf{b}$, under the typical experimental reaction conditions mentioned above, led to the formation of the corresponding 2-(arylhydrazono)-1,4-dihydro-naphtho[1,8-ef][1,4]diazepine-3-ones 5a,b. The structure elucidation of the reaction products $\mathbf{5 a}, \mathbf{b}$ was based on their elemental analyses and spectral data (IR, MS and 
$\left.{ }^{1} \mathrm{H}-\mathrm{NMR}\right)$. The IR spectra of the reaction products exhibited three $\mathrm{NH}$ absorptions in the $3,325-3,120 \mathrm{~cm}^{-1}$ region in addition to an amide $-\mathrm{C}=\mathrm{O}$ absorption band around $1,660 \mathrm{~cm}^{-1}$, as well as three singlet $\mathrm{D}_{2} \mathrm{O}$ exchangeable-NH proton signals resonating in the 9.95-10.91 region in their ${ }^{1} \mathrm{H}-\mathrm{NMR}$ spectra. Moreover, their mass spectra revealed in each case a peak due to the molecular ion $\left(\mathrm{M}^{+}\right)$. The electronic absorption spectra data of $\mathbf{5}$ in dioxane revealed, in each case, one characteristic absorption band in the 378 and $385 \mathrm{~nm}$ region corresponding to the hydrazone chromophore $\mathbf{5 A}[33,34]$ (Table 1).

Scheme 1. Reaction of 1,8-diaminonaphthalene (1) with hydrazonoyl chlorides 2 and 4.

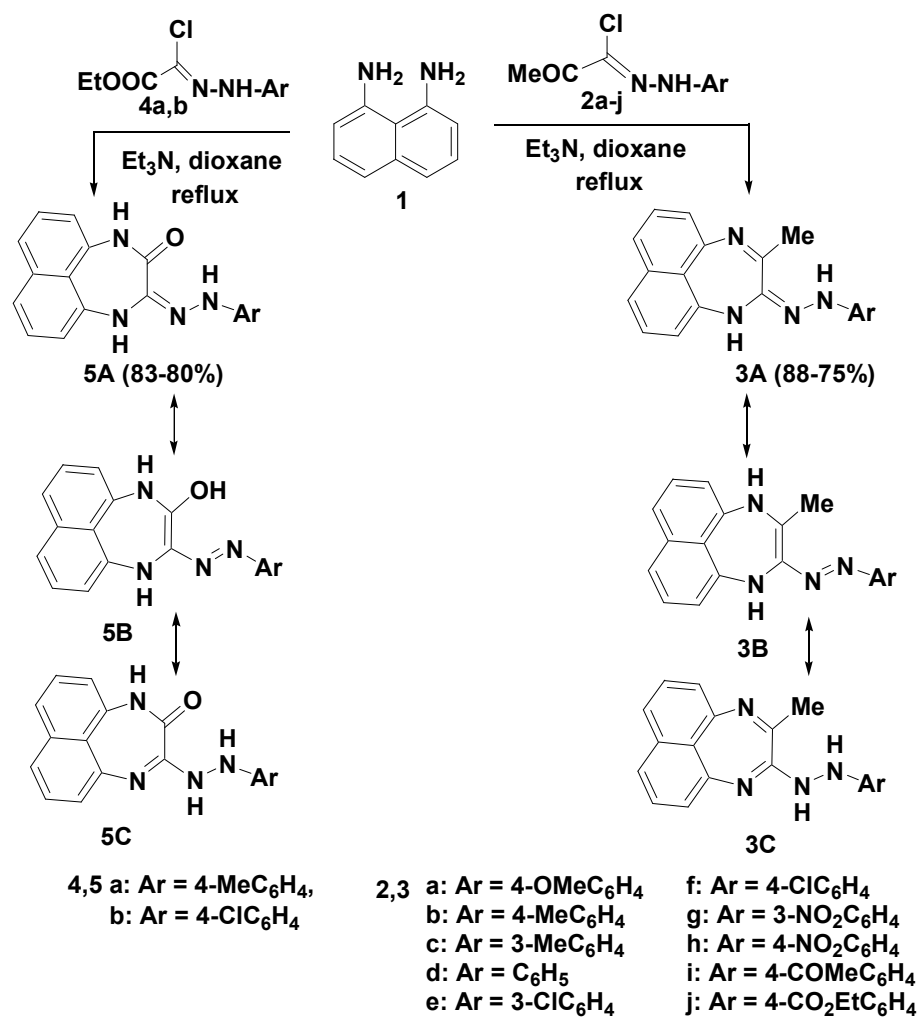

Table 1. UV Spectral data of compounds $3 \mathbf{a}-\mathbf{j}, \mathbf{5 a}, \mathbf{b}$ and $\mathbf{1 1 a}-\mathbf{h}$ in dioxane.

\begin{tabular}{cccc}
\hline Compd. No. & $\boldsymbol{\lambda}_{\max }(\log \boldsymbol{\varepsilon})$ & Compd. No. & $\boldsymbol{\lambda}_{\max }(\log \boldsymbol{\varepsilon})$ \\
\hline $\mathbf{3 a}$ & $359(4.25)$ & $\mathbf{5 a}$ & $378(4.45)$ \\
$\mathbf{3 b}$ & $349(3.89)$ & $\mathbf{5 b}$ & $385(4.33)$ \\
$\mathbf{3 c}$ & $347(3.59)$ & $\mathbf{1 1 a}$ & $484(4.24), 346(4.19), 269(4.24)$ \\
$\mathbf{3 d} *$ & $348(4.30)$ & $\mathbf{1 1 b}$ & $476(4.01), 340(4.10), 233(4.28)$ \\
$\mathbf{3 e}$ & $349(3.87)$ & $\mathbf{1 1 c} * *$ & $473(4.15), 351(4.32), 231(4.38)$ \\
$\mathbf{3 f}$ & $349(3.88)$ & $\mathbf{1 1 d}$ & $472(4.24), 339(4.15), 232(4.35)$ \\
$\mathbf{3 g}$ & $345(3.89)$ & $\mathbf{1 1 e}$ & $472(4.0), 337(3.89), 267(4.01)$ \\
$\mathbf{3 h}$ & $381(3.76)$ & $\mathbf{1 1 f}$ & $490(4.66), 357(4.57), 231(4.89)$ \\
$\mathbf{3 i}$ & $359(4.19)$ & $\mathbf{1 g}$ & $509(4.05), 361(4.11), 262(4.13)$ \\
$\mathbf{3 j}$ & $351(4.05)$ & $\mathbf{1 1 h}$ & $478(4.10), 336(3.79), 296(3.80)$ \\
\hline
\end{tabular}

* Solvent $\lambda_{\max }(\log \varepsilon)$ : acetone: 349 (4.01); chloroform: 348 (4.02); cyclohexane: 347 (4.11); ethanol 348 (4.04); DMF 349 (4.24); ${ }^{* *}$ Solvent $\lambda_{\max }(\log \varepsilon)$ : acetone: 473 (4.20), 350 (4.35), 231 (4.41); chloroform: 473 (3.99), 353 (4.15), 233 (4.28); cyclohexane: 473 (4.25), 350 (4.10), 230 (4.08); ethanol 472 (4.24), 352 (4.23), 236 (4.47); DMF 476 (4.36), 346 (4.29), 231 (4.38). 
From the literature [35,36], we found that reaction of 4-hydroxycoumarin with 1,2-phenylenediamine furnished 1,5-benzodiazepin-2-ones. This finding attracted our intention to study the reaction of 4-hydroxycoumarin with 1,8-diaminonaphthalene. Thus, heating a 1:1 molar mixture of 1,8-diaminonaphthalene (1) with 4-hydroxycoumarin (6) in refluxing ethanol furnished a single product for which two possible structures; the naphtho[1,8-bc][1,5]diazocin-2-one structure 7 or the 1,2-dihydroperimidine 8 derivatives can be postulated (Scheme 2). Based on its spectral data (MS, IR, ${ }^{1} \mathrm{H}-\mathrm{NMR}$ and $\left.{ }^{13} \mathrm{C}-\mathrm{NMR}\right)$ the 2-(2-hydroxybenzoylmethylene)-1,2-dihydroperimidine structure 8 was assigned to the reaction product. For example, its ${ }^{13} \mathrm{C}$-NMR revealed a characteristic signal at $\delta 186.22$ due to a ketonic carbonyl-carbon rather than an amidic carbonyl-carbon where the latter one resonates in the region $\delta 160-166$ [25] and the signal for methene carbon at 77.15 [37]. Also, the carbon signals of the perimidine moiety were clearly observed in the ${ }^{13} \mathrm{C}$-NMR spectrum of compound 8 [37]. Further evidence for the assigned structure $\mathbf{8}$ is provided by its mass spectrum, which revealed ion peaks at $m / z$ 168 and 121 , corresponding to the perimidine and $\mathrm{HO}-\mathrm{C}_{6} \mathrm{H}_{4}-\mathrm{CO}$ fragments, respectively. This assignment is in good agreement with literature data indicating the stability of perimidines to be greater than that of diazocines [37-39].

Scheme 2. Reaction of 1,8-diaminonaphthalene (1) with 4-hydroxycoumarin (6).

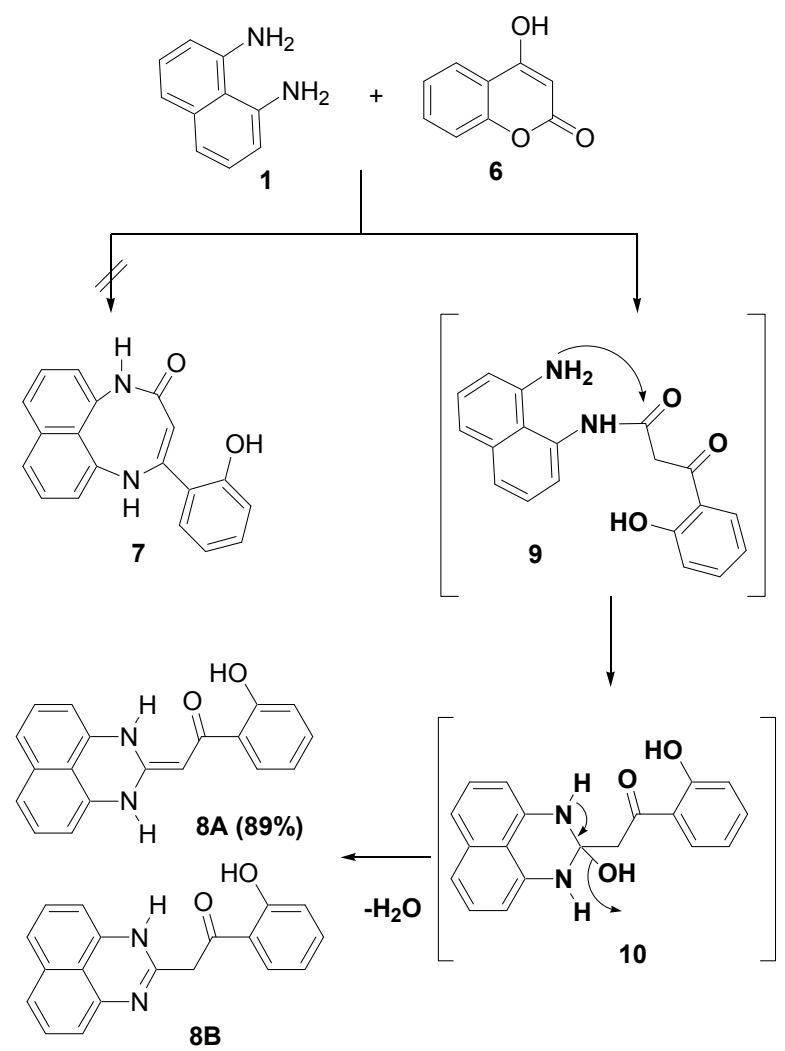

To account for the formation of the product $\mathbf{8}$, it is suggested, as depicted in Scheme 2, that the reaction of $\mathbf{1}$ with $\mathbf{6}$ starts with two nucleophilic addition reactions to give the intermediates $\mathbf{9}$ and $\mathbf{1 0}$ which underwent in situ cyclization via elimination of water molecules to give $\mathbf{8}$ as the end product. Compound 8 has two possible tautomeric forms, $\mathbf{8 A}$ and $\mathbf{8 B}$, however the presence of a singlet signal resonating at 5.62 integrated for $1 \mathrm{H}$ due to $(-\mathrm{C}=\mathrm{CH}-)$ and the absence of any aliphatic protons in the 2-4 region of the ${ }^{1} \mathrm{H}-\mathrm{NMR}$ confirmed the tautomer $\mathbf{8 A}$ as the main product (Scheme 2). 
The reactivity of the multifunctional -enaminoketone $\mathbf{8 A}$ towards the hydrazonoyl chlorides $\mathbf{4}$ was also investigated. Both the -enaminoketone 8A and hydrazonoyl chlorides $\mathbf{4}$ have reaction different sites that may attack each other, thus, when compound $\mathbf{8 A}$ was treated with the ester hydrazonoyl chlorides $\mathbf{4 a}-\mathbf{h}$ in refluxing dioxane in the presence of triethylamine, it afforded, in each case, only one isolable product whose elemental analyses and mass spectral data were consistent with compounds 11, 12 or 13 (Scheme 3), where all products have identical molecular ion peaks due to elimination of $\mathrm{HCl}$ and ethanol molecules.

Scheme 3. Reaction of compound $\mathbf{8}$ with hydrazonoyl chlorides $\mathbf{4 a}-\mathbf{h}$.

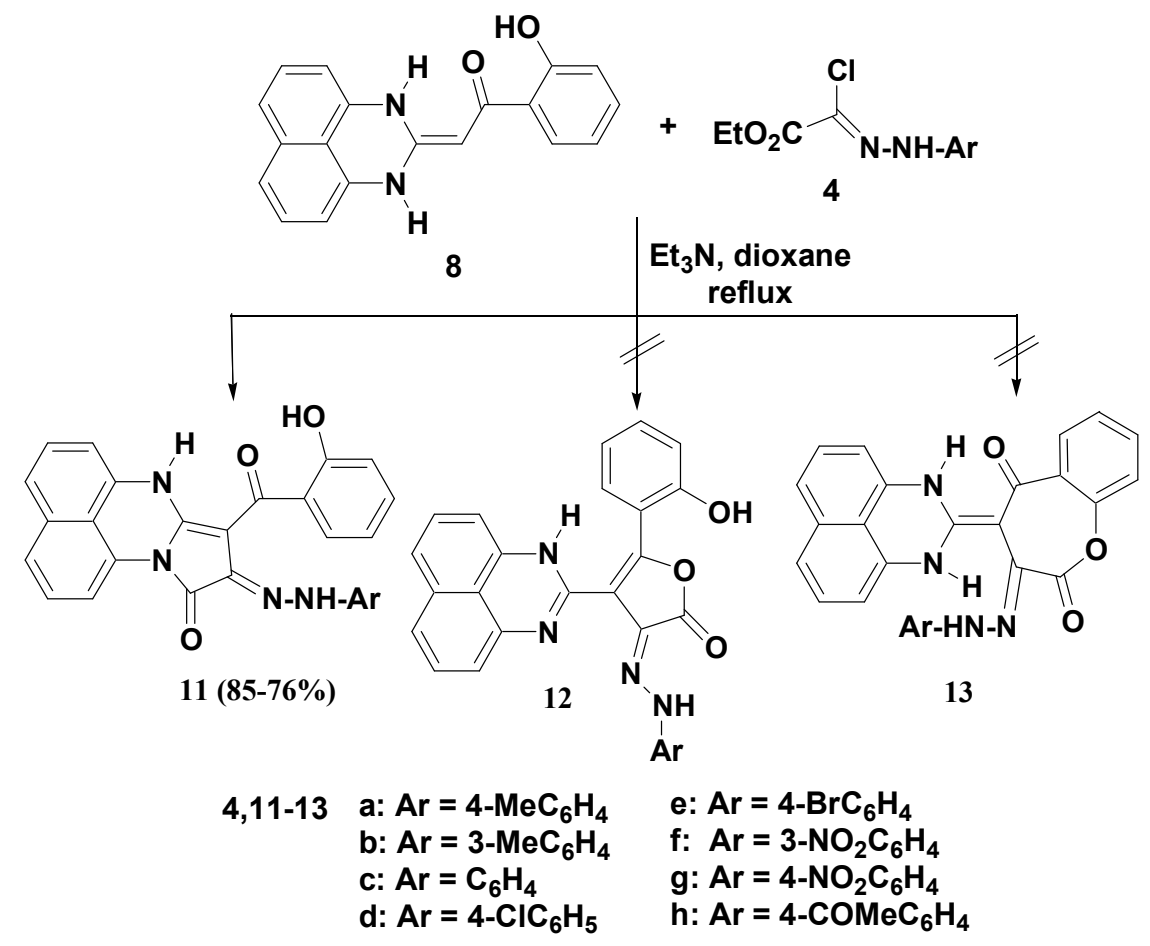

The IR spectra of the products in the solid state showed carbonyl group bands in the $1693-1687 \mathrm{~cm}^{-1}$ (cyclic amide $\mathrm{C}=\mathrm{O}$ group) and $1647-1639 \mathrm{~cm}^{-1}$ (benzoyl $\mathrm{C}=\mathrm{O}$ group) regions, which are consistent with the structure of compound $\mathbf{1 1}$ rather than its isomeric structures $\mathbf{1 2}$ or $\mathbf{1 3}$ (Scheme 3). The mechanism outlined in Scheme 4 accounts for the formation of compound 11. Due to the conjugation effect of the electron-donating amino group of compound $\mathbf{4}$ and the electron-withdrawing substituent (2-hydroxybenzoyl group), the double bond is highly polarized and the electron density on the $\alpha$-carbon is greater than that of the nitrogen atom so the reaction is assumed to proceed through carbon-carbon cross-coupling via initial attack of the activated -enamine's carbon (nucleophile) of $\mathbf{8 A}$ on the electrophilic carbon of nitrilimine $\mathbf{4 A}$, followed by elimination of ethanol to give the desired product 11 as depicted in Scheme 4. As products 11 can have five possible tautomeric structures $11 \mathbf{A}-\mathbf{E}$, their electronic absorption spectra were studied in dioxane and showed in each case three absorption bands in the 509-472, 361-336 and 296-231 nm regions (Figure 1). This absorption pattern is similar to that reported for analogous azo chromophore [40]. Such data indicate that the actual tautomeric structure of such compounds in solution phase is the arylazohydroxy form 11B, whereas their IR spectral data indicate that they exist in the solid state as the diketo-hydrazone tautomeric form 11A (Figure 1). 
Scheme 4. The mechanism of formation of compounds 11.

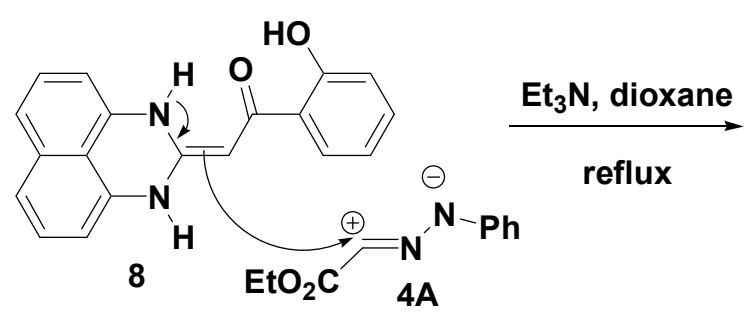

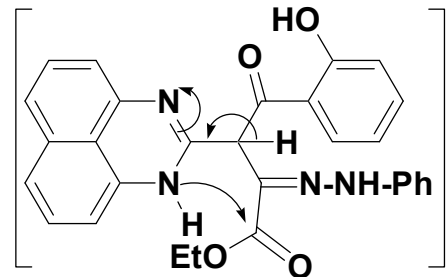

14

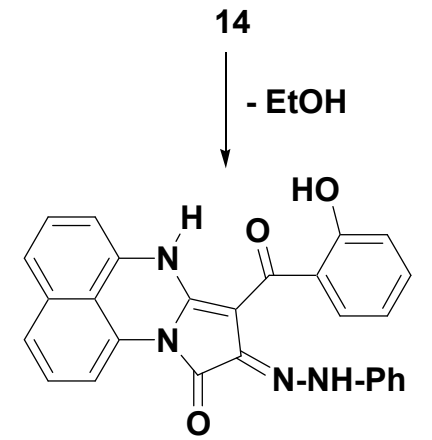

11

Figure 1. Tautomeric structures of compounds 11.
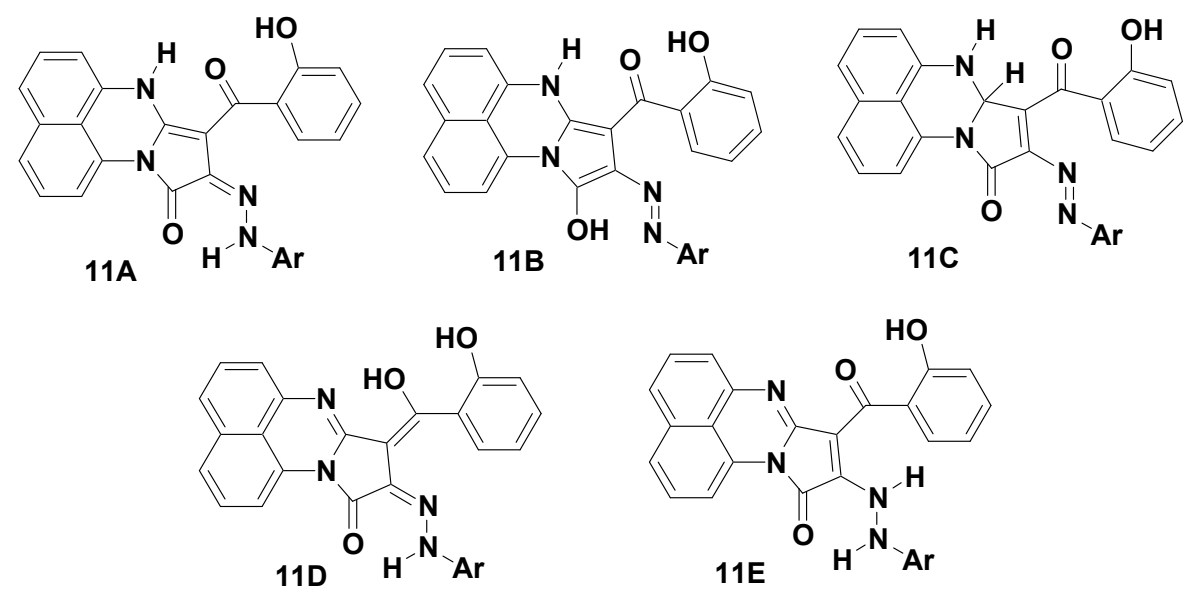

\subsection{Results of Biological Activity}

\subsubsection{Effect on the Growth of Human Cancer Cell Lines}

The effects of the 16 newly synthesized compounds $\mathbf{3 c}-\mathbf{f}, \mathbf{3 h}-\mathbf{j}, \mathbf{8}, \mathbf{1 1 a}-\mathbf{h}$ on the growth of three human cancer cell lines, TK-10, MCF-7 and UACC-62, given in concentrations that were able to cause $50 \%$ of cell growth inhibition, are summarized in Table 2 . Regarding the activities of the naphtha[1,8ef]diazepine derivatives $\mathbf{3}$, no capacity to inhibit the growth of the human cancer cell lines was observed with $\mathbf{3 e}$ and $\mathbf{3 h}$, while compound $\mathbf{3 i}$ inhibited the $\mathrm{GI}_{50}$ on all the cell lines (TK-10 $=96.73 \mu \mathrm{M} / \mathrm{mL}$, $\mathrm{MCF}-7=81.29 \mu \mathrm{M} / \mathrm{mL}$ and UACC-62 $=41.97 \mu \mathrm{M} / \mathrm{mL})$. Compounds $\mathbf{3 c}$ and $\mathbf{3} \mathbf{j}$ showed an inhibitory effect against the growth of the TK-10 and MCF-7 cells, but did not possess any activity on the UACC-62 cell line. Compounds 3d and 3f showed an inhibitory effect against one cell line only (MCF-7 and TK-10 cells, respectively). On the other hand, regarding the activities of the pyrrolo[1,2$\alpha$ perimidine derivatives $\mathbf{1 1 a}-\mathbf{h}$, compound $11 \mathrm{c}$ showed growth inhibitory effects that were stronger to 
TK-10 and MCF-7 cell lines and its inhibitory parameters $\left(\mathrm{GI}_{50}\right.$, TGI and $\left.\mathrm{LC}_{50}\right)$ demonstrated total growth inhibition (TGI $=25.28,21.87 \mu \mathrm{M} / \mathrm{mL}$, respectively) compared to those presented by the rest of the group which affected only on one or two of the experimented cell lines. Compound $\mathbf{8}$ showed an inhibitory effect against the growth of the TK-10 and MCF-7 cells, while the other derivatives 11a,b,e-h showed no activities.

Table 2. Concentration ( $\mu \mathrm{M} / \mathrm{mL})$ required to inhibit cell growth by $50 \%\left(\mathrm{GI}_{50}\right)$, to produce total growth inhibition (TGI) and to cause $50 \%$ net cell killing $\left(\mathrm{LC}_{50}\right)$.

\begin{tabular}{|c|c|c|c|c|}
\hline Product & Inhibition parameters & TK-10 & MCF-7 & UACC-62 \\
\hline \multirow{3}{*}{ 3c } & $\mathrm{GI}_{50}$ & $62.44 \pm 3.58$ & $80.66 \pm 5.24$ & $>100$ \\
\hline & TGI & $>100$ & $>100$ & $>100$ \\
\hline & $\mathrm{LC}_{50}$ & $>100$ & $>100$ & $>100$ \\
\hline \multirow{3}{*}{ 3d } & $\mathrm{GI}_{50}$ & $>100$ & $95.48 \pm 11.62$ & $>100$ \\
\hline & TGI & $>100$ & $>100$ & $>100$ \\
\hline & $\mathrm{LC}_{50}$ & $>100$ & $>100$ & $>100$ \\
\hline \multirow{3}{*}{$3 f$} & $\mathrm{GI}_{50}$ & $83.72 \pm 9.73$ & $>100$ & $>100$ \\
\hline & TGI & $>100$ & $>100$ & $>100$ \\
\hline & $\mathrm{LC}_{50}$ & $>100$ & $>100$ & $>100$ \\
\hline \multirow{3}{*}{$3 \mathbf{i}$} & $\mathrm{GI}_{50}$ & $96.73 \pm 11.27$ & $81.29 \pm 8.28$ & $41.97 \pm 4.97$ \\
\hline & TGI & $>100$ & $>100$ & $86.66 \pm 7.14$ \\
\hline & $\mathrm{LC}_{50}$ & $>100$ & $>100$ & $>100$ \\
\hline \multirow{3}{*}{$\mathbf{3 j}$} & $\mathrm{GI}_{50}$ & $71.06 \pm 4.87$ & $63.87 \pm 3.49$ & $>100$ \\
\hline & TGI & $>100$ & $>100$ & $>100$ \\
\hline & $\mathrm{LC}_{50}$ & $>100$ & $>100$ & $>100$ \\
\hline \multirow{3}{*}{8} & $\mathrm{GI}_{50}$ & $72.08 \pm 6.94$ & $42.25 \pm 4.11$ & $>100$ \\
\hline & TGI & $>100$ & $>100$ & $>100$ \\
\hline & $\mathrm{LC}_{50}$ & $>100$ & $>100$ & $>100$ \\
\hline \multirow{3}{*}{ 11c } & $\mathrm{GI}_{50}$ & $16.39 \pm 1.79$ & $12.67 \pm 1.08$ & $43.78 \pm 1.05$ \\
\hline & TGI & $25.28 \pm 2.39$ & $21.87 \pm 2.10$ & $>100$ \\
\hline & $\mathrm{LC}_{50}$ & $38.99 \pm 3.01$ & $37.77 \pm 3.91$ & $>100$ \\
\hline
\end{tabular}

The range of doses assayed was $0.01-100 \mu \mathrm{M} / \mathrm{mL}$; Results are mean $\pm \mathrm{SE}$ (standard error of the mean) $(n=3)$; The $\mathrm{GI}_{50}$, TGI and $\mathrm{LC}_{50}$ for the other products are $>100$.

In conclusion, concerning the effect on the growth of human cancer cell lines, 11c was found to have the best inhibitory activity towards all the three cell lines, followed by $3 \mathbf{i}$ that caused $50 \%$ cell growth inhibition on all the cancer cell lines. This activity could be attributed to heterocyclic fused rings system such as naphtha[1,8-ef]diazepines and pyrrolo[1,2-a]perimidine derivatives containing multiple centers with hydrogen accepting properties (COMe, $\mathrm{C}=\mathrm{O}$ and nitrogen atoms), are essential for DNA intercalating activity and antitumor activity.

\subsubsection{Oxygen Radical Absorbance Capacity (ORAC) Assay}

All the samples show weak antioxidant (ORAC) activity as scavengers. Compounds with antioxidant potential can directly interact with ROS, thereby reducing oxidative damage [41]. To investigate further the mechanism of action by which compounds $\mathbf{3}, \mathbf{8}$ and $\mathbf{1 1}$ exert a protective effect 
under oxidative stress conditions, the potential radical-scavenging activity using the oxygen radical absorbance capacity (ORAC) chemical assay was evaluated.

The values of the area under the curve (AUC, Table 3) showed also that compounds $\mathbf{3 ,} \mathbf{8}$ and $\mathbf{1 1}$ are much weaker scavengers than the reference compound Trolox, as the decline in fluorescence over time was significantly more rapid for all of the tested compounds than for Trolox (assayed at $8 \mu \mathrm{M}$ concentration), indicating that they are not as effective as Trolox in scavenging peroxyl radicals generated by the thermal decomposition of 2,2'-azobis(2-amidinopropane) dihydrochloride (AAPH). Thus, because peroxy radicals are not effectively neutralized by the studied compounds, fluorescein is degraded, resulting in non-fluorescent products.

Table 3. ORAC values for antioxidant activity of compounds 3, 8, and $\mathbf{1 1 .}$

\begin{tabular}{cccc}
\hline Comp. No. & ORAC Value & Comp. No. & ORAC Value \\
\hline 3d & $0.61 \pm 0.04$ & $\mathbf{1 1 c}$ & $0.86 \pm 0.09$ \\
$\mathbf{3 f}$ & $0.74 \pm 0.07$ & $\mathbf{1 1 d}$ & $0.43 \pm 0.59$ \\
$\mathbf{3 h}$ & $0.54 \pm 0.06$ & $\mathbf{1 1 b}$ & $0.51 \pm 0.10$ \\
$\mathbf{3 i}$ & $0.97 \pm 0.10$ & $\mathbf{1 1 f}$ & $0.60 \pm 0.07$ \\
$\mathbf{3 j}$ & $0.68 \pm 0.03$ & $\mathbf{1 1 e}$ & $0.62 \pm 0.09$ \\
$\mathbf{3 c}$ & $0.81 \pm 0.05$ & $\mathbf{1 1 g}$ & $0.42 \pm 0.05$ \\
$\mathbf{3 e}$ & $0.65 \pm 0.07$ & $\mathbf{1 1 a}$ & $0.53 \pm 0.08$ \\
$\mathbf{8}$ & $0.72 \pm 0.06$ & $\mathbf{1 1 h}$ & $0.59 \pm 0.06$ \\
\hline
\end{tabular}

Data expressed as mean \pm SE (standard error of the mean) $(n=3)$, and ORAC values expressed as $\mu \mathrm{M}$ of Trolox/mg sample.

\section{Experimental}

\subsection{General Information}

All melting points were determined on an electrothermal Gallenkamp apparatus. Solvents were generally distilled and dried by standard literature procedures prior to use. The IR spectra were measured on a Pye-Unicam $\mathrm{SP}_{300}$ instrument in potassium bromide discs. The ${ }^{1} \mathrm{H}-\mathrm{NMR}$ spectra were recorded on a Varian Mercury VXR-300 MHz spectrometer (300 MHz for ${ }^{1} \mathrm{H}-\mathrm{NMR}$ and $75 \mathrm{MHz}$ for ${ }^{13} \mathrm{C}-\mathrm{NMR}$ ) and reported as the chemical shifts $\delta(\mathrm{ppm})$ downfield from tetramethylsilane (TMS) used as an internal standard. The mass spectra were recorded on a GCMS-Q1000-EX Shimadzu or GCMS 5988-A HP spectrometer; the ionizing voltage was $70 \mathrm{eV}$. Elemental analyses were carried out by the Microanalytical Center of Cairo University, Giza, Egypt. TLC was run on silica gel G coated plates and iodine vapour was used as visualizing agent.

\subsection{General Procedure for the Reaction of Hydrazonoyl Chlorides with 1,8-Diaminonaphthalene}

To a stirred solution of 1,8-diaminonaphthalene (1, $0.79 \mathrm{~g}, 5 \mathrm{mmol})$ and the appropriate hydrazonoyl chloride $(5 \mathrm{mmol})$ in dioxane $(40 \mathrm{~mL})$, triethylamine $(0.7 \mathrm{~mL})$ was added dropwise and the mixture was then refluxed for $5 \mathrm{~h}$. The precipitated triethylamine hydrochloride was filtered off and the filtrate was evaporated under reduced pressure. The residue was triturated with methanol. The solid product formed in each case was collected by filtration, washed with water, dried and 
recrystallized from a suitable solvent system as indicated in each example to afford the corresponding derivatives $\mathbf{3 a}-\mathbf{j}$ and $\mathbf{5 b}, \mathbf{d}, \mathbf{f}$.

2-(4-Methoxyphenylazo)-3-methyl-1H-naphtho[1,8-ef][1,4]diazepine (3a). Red solid (86\%); mp 200-202 ${ }^{\circ} \mathrm{C}(\mathrm{EtOH})$; IR (KBr) $v_{\max } 3394,3286(2 \mathrm{NH}) \mathrm{cm}^{-1} ;{ }^{1} \mathrm{H}-\mathrm{NMR}$ (DMSO-d $) \delta 2.25\left(\mathrm{~s}, 3 \mathrm{H}, \mathrm{CH}_{3}\right)$, $3.58\left(\mathrm{~s}, 3 \mathrm{H}, \mathrm{OCH}_{3}\right), 6.65-7.92(\mathrm{~m}, 10 \mathrm{H}, \mathrm{Ar}-\mathrm{H}), 9.98\left(\mathrm{~s}, 1 \mathrm{H}, \mathrm{NH}, \mathrm{D}_{2} \mathrm{O}\right.$-exchangeable), $10.10(\mathrm{~s}, 1 \mathrm{H}$, $\mathrm{NH}, \mathrm{D}_{2} \mathrm{O}$-exchangeable); MS m/z (\%) $331\left(\mathrm{M}^{+}+1,4\right), 330\left(\mathrm{M}^{+}, 14\right), 327$ (5), 140 (12), 123 (18), 122 (100), 107 (11), 77 (6). Anal. Calcd. for $\mathrm{C}_{20} \mathrm{H}_{18} \mathrm{~N}_{4} \mathrm{O}$ (330.38): C, 72.71; H, 5.49; N, 16.96. Found: C, $72.49 ; \mathrm{H}, 5.37 ; \mathrm{N}, 16.75 \%$.

2-(4-Methylphenylazo)-3-methyl-1H-naphtho[1,8-ef][1,4]diazepine (3b). Orange solid (88\%); mp 240-242 ${ }^{\circ} \mathrm{C}(\mathrm{EtOH})$; IR (KBr) $v_{\max } 3398,3105(2 \mathrm{NH}) \mathrm{cm}^{-1} ;{ }^{1} \mathrm{H}-\mathrm{NMR}$ (DMSO-d $) \delta 2.28\left(\mathrm{~s}, 3 \mathrm{H}, \mathrm{CH}_{3}\right)$, 2.35 (s, 3H, $\left.\mathrm{CH}_{3}\right), 7.13-7.55$ (m, 10H, Ar-H), 10.63 (s, 1H, NH, D $\mathrm{D}_{2} \mathrm{O}$-exchangeable), $12.01(\mathrm{~s}, 1 \mathrm{H}$, $\mathrm{NH}, \mathrm{D}_{2} \mathrm{O}$-exchangeable); MS m/z (\%) $315\left(\mathrm{M}^{+}+1,24\right), 314\left(\mathrm{M}^{+}, 100\right), 313$ (10), 297 (14), 209 (33), 167 (64), 140 (69), 106 (94), 91 (23), 77 (45). Anal. Calcd. for $\mathrm{C}_{20} \mathrm{H}_{18} \mathrm{~N}_{4}$ (314.38): C, 76.41; H, 5.77; N, 17.82. Found: C, 76.26; H, 5.47; N, 17.61\%.

2-(3-Methylphenylazo)-3-methyl-1H-naphtho[1,8-ef][1,4]diazepine (3c). Orange solid (78\%); mp $150-152{ }^{\circ} \mathrm{C}(\mathrm{EtOH})$; IR (KBr) $v_{\max } 3345,3225(2 \mathrm{NH}) \mathrm{cm}^{-1} ;{ }^{1} \mathrm{H}-\mathrm{NMR}$ (DMSO-d $\left.)_{6}\right) 2.25(\mathrm{~s}, 3 \mathrm{H}$, $\left.\mathrm{CH}_{3}\right), 2.32$ (s, 3H, $\left.\mathrm{CH}_{3}\right), 6.85-7.37(\mathrm{~m}, 10 \mathrm{H}, \mathrm{Ar}-\mathrm{H}), 9.85$ (s, 1H, NH, $\mathrm{D}_{2} \mathrm{O}$ exchangeable), 10.20 (s, $1 \mathrm{H}, \mathrm{NH}, \mathrm{D}_{2} \mathrm{O}$ exchangeable); MS m/z (\%), $315\left(\mathrm{M}^{+}+1,24\right), 314\left(\mathrm{M}^{+}, 100\right), 313$ (72), 297 (20), 280 (13), 208 (8), 167 (71), 147 (66), 140 (70), 107 (52), 91 (19), 77 (29). Anal. Calcd. for $\mathrm{C}_{20} \mathrm{H}_{18} \mathrm{~N}_{4}$ (314.38): C, 76.41; H, 5.77; N, 17.82. Found: C, 76.24; H, 5.51; N, 17.64\%.

2-Phenylazo-3-methyl-1H-naphtho[1,8-ef][1,4]diazepine (3d). Red solid (86\%); mp 194-196 ${ }^{\circ} \mathrm{C}$ (EtOH); IR (KBr) $v_{\max } 3215,3154(2 \mathrm{NH}) \mathrm{cm}^{-1}$; ${ }^{1} \mathrm{H}-\mathrm{NMR}\left(\mathrm{DMSO}-d_{6}\right) \delta 2.21\left(\mathrm{~s}, 3 \mathrm{H}, \mathrm{CH}_{3}\right)$, 6.66-7.53 (m, 11H, Ar-H), 9.67 (s, 1H, NH, $\mathrm{D}_{2} \mathrm{O}$-exchangeable), 9.90 (s, 1H, NH, $\mathrm{D}_{2} \mathrm{O}$-exchangeable); MS m/z (\%) $300\left(\mathrm{M}^{+}, 45\right), 299$ (35), 283 (17), 255 (21), 251 (20), 207 (41), 166 (100), 89 (21), 77 (21). Anal. Calcd. for $\mathrm{C}_{19} \mathrm{H}_{16} \mathrm{~N}_{4}$ (300.36): C, 75.98; H, 5.37; N, 18.65. Found: C, 75.84; H, 5.21; N, 18.46\%.

2-(3-Chlorophenylazo)-3-methyl-1H-naphtho[1,8-ef][1,4]diazepine (3e). Orange solid (75\%); mp 230-232 ${ }^{\circ} \mathrm{C}(\mathrm{EtOH})$; IR (KBr) $v_{\max } 3200,3116(2 \mathrm{NH}) \mathrm{cm}^{-1} ;{ }^{1} \mathrm{H}-\mathrm{NMR}$ (DMSO-d $) \delta 2.31\left(\mathrm{~s}, 3 \mathrm{H}, \mathrm{CH}_{3}\right)$, 7.01-7.89 (m, 10H, Ar-H), 10.20 (s, 1H, NH, D $2 \mathrm{O}$-exchangeable), 10.54 (s, 1H, NH, $\mathrm{D}_{2} \mathrm{O}$-exchangeable); MS m/z (\%) $336\left(\mathrm{M}^{+}+2,7\right), 335\left(\mathrm{M}^{+}+1,8\right), 334\left(\mathrm{M}^{+}, 31\right), 208(20), 166(75), 140$ (100), 111 (12), 90 (12), 75 (16). Anal. Calcd. for $\mathrm{C}_{19} \mathrm{H}_{15} \mathrm{ClN}_{4}$ (334.80): C, 68.16; H, 4.52; N, 16.73. Found: C, 68.05; H, 4.34; N, 16.41\%.

2-(4-Chlorophenylazo)-3-methyl-1H-naphtho[1,8-ef][1,4]diazepine (3f). Deep-orange solid (83\%); $\mathrm{mp}>300{ }^{\circ} \mathrm{C}\left(\right.$ EtOH/dioxane); IR (KBr) $v_{\max } 3325,3150(2 \mathrm{NH}) \mathrm{cm}^{-1} ;{ }^{1} \mathrm{H}-\mathrm{NMR}$ (DMSO-d $) \delta 2.27$ (s, $\left.3 \mathrm{H}, \mathrm{CH}_{3}\right), 6.76-7.58(\mathrm{~m}, 10 \mathrm{H}, \mathrm{Ar}-\mathrm{H}), 9.91\left(\mathrm{~s}, 1 \mathrm{H}, \mathrm{NH}, \mathrm{D}_{2} \mathrm{O}\right.$ exchangeable), 10.01 (s, 1H, NH, $\mathrm{D}_{2} \mathrm{O}$ exchangeable); MS m/z (\%) $336\left(\mathrm{M}^{+}+2,19\right), 335\left(\mathrm{M}^{+}+1,21\right), 334\left(\mathrm{M}^{+}, 60\right), 208$ (13), $166(100), 139$ (19), 126 (12), 113 (10), 111 (4), 63 (11). Anal. Calcd. For $\mathrm{C}_{19} \mathrm{H}_{15} \mathrm{ClN}_{4}$ (334.80): C, 68.16; H, 4.52; N, 16.73. Found: C, 68.05; H, 4.34; N, 16.41\%. 
2-(3-Nitrophenylazo)-3-methyl-1H-naphtho[1,8-ef] [1,4]diazepine (3g). Green solid (77\%); mp > $300{ }^{\circ} \mathrm{C}$ (EtOH/dioxane); IR (KBr) $v_{\max } 3348,3100(2 \mathrm{NH}) \mathrm{cm}^{-1} ;{ }^{1} \mathrm{H}-\mathrm{NMR}$ (DMSO- $\left.d_{6}\right) \delta 2.31\left(\mathrm{~s}, 3 \mathrm{H}, \mathrm{CH}_{3}\right)$, 6.89-8.07 (m, 10H, Ar-H), $8.31\left(\mathrm{~s}, 1 \mathrm{H}, \mathrm{NH}, \mathrm{D}_{2} \mathrm{O}\right.$-exchangeable), $10.60\left(\mathrm{~s}, 1 \mathrm{H}, \mathrm{NH}, \mathrm{D}_{2} \mathrm{O}\right.$ exchangeable); MS m/z (\%) 345 (M+, 68), 328 (4), 234 (8), 208 (19), 194 (15), 166 (100), 140 (93), 76 (6). Anal. Calcd. for $\mathrm{C}_{19} \mathrm{H}_{15} \mathrm{~N}_{5} \mathrm{O}_{2}$ (345.35): C, 66.08; H, 4.38; N, 20.28. Found: C, 65.91; H, 4.11; N, 20.04\%.

2-(4-Nitrophenylazo)-3-methyl-1H-naphtho[1,8-ef] [1,4]diazepine (3h). Pale-brown solid (75\%); mp 260-262 ${ }^{\circ} \mathrm{C}\left(\right.$ EtOH/dioxane); IR (KBr) $v_{\max } 3382,3290(2 \mathrm{NH}) \mathrm{cm}^{-1} ;{ }^{1} \mathrm{H}-\mathrm{NMR}$ (DMSO- $\left.d_{6}\right) \delta 2.26$ (s, $\left.3 \mathrm{H}, \mathrm{CH}_{3}\right), 6.66-7.17$ (m, 6H, Ar-H), 7.66 (d, $\left.J=9 \mathrm{~Hz}, 2 \mathrm{H}, \mathrm{Ar}-\mathrm{H}\right), 8.17$ (d, $\left.J=9 \mathrm{~Hz}, 2 \mathrm{H}, \mathrm{Ar}-\mathrm{H}\right), 10.03$ (s, 1H, NH, $\mathrm{D}_{2} \mathrm{O}$-exchangeable), 10.43 (s, 1H, NH, $\mathrm{D}_{2} \mathrm{O}$-exchangeable); $\mathrm{MS} m / z(\%) 345\left(\mathrm{M}^{+}, 69\right), 344$ (54), 342 (43), 208 (29), 166 (100), 140 (57), 122 (20), 114 (46), 108 (46), 77 (26), 60 (63). Anal. Calcd. for $\mathrm{C}_{19} \mathrm{H}_{15} \mathrm{~N}_{5} \mathrm{O}_{2}$ (345.35): C, 66.08; H, 4.38; N, 20.28. Found: C, 65.87; H, 4.20; N, 20.12\%.

2-(4-Acetylphenylazo)-3-methyl-1H-naphtho[1,8-ef][1,4]diazepine (3i). Dark orange solid (78\%); mp 280-282 ${ }^{\circ} \mathrm{C}\left(\right.$ EtOH/dioxane); IR (KBr) v $v_{\max } 3394,3286(2 \mathrm{NH}), 1654(\mathrm{C}=\mathrm{O}) \mathrm{cm}^{-1}$; ${ }^{1} \mathrm{H}-\mathrm{NMR}$ $\left(\mathrm{DMSO}-d_{6}\right) \delta 2.25\left(\mathrm{~s}, 3 \mathrm{H}, \mathrm{CH}_{3}\right), 2.51\left(\mathrm{~s}, 3 \mathrm{H}, \mathrm{CH}_{3}\right), 6.65-7.20(\mathrm{~m}, 6 \mathrm{H}, \mathrm{Ar}-\mathrm{H}), 7.59(\mathrm{~d}, J=9 \mathrm{~Hz}, 2 \mathrm{H}$, Ar-H), 7.89 (d, $J=9 \mathrm{~Hz}, 2 \mathrm{H}, \operatorname{Ar}-\mathrm{H}), 9.98$ (s, 1H, NH, $\mathrm{D}_{2} \mathrm{O}$-exchangeable), 10.10 (s, 1H, NH, $\mathrm{D}_{2} \mathrm{O}$-exchangeable); MS m/z (\%) 342 (M+ 58), 341 (48), 208 (12), 166 (100), 163 (22), 140 (56), 139 (43), 120 (14), 91 (10). Anal. Calcd. for $\mathrm{C}_{21} \mathrm{H}_{18} \mathrm{~N}_{4} \mathrm{O}$ (342.39): C, 73.67; H, 5.30; N, 16.36. Found: C, $73.52 ; \mathrm{H}, 5.17 ; \mathrm{N}, 16.15 \%$.

2-(4-Ethoxycarbonylphenylhydrazono)-3-methyl-1H-naphtho[1,8-ef][1,4] diazepine (3j). Red solid (81\%); mp 120-122 ${ }^{\circ} \mathrm{C}(\mathrm{EtOH}) ; \mathrm{IR}(\mathrm{KBr}) v_{\max } 3200,3150(2 \mathrm{NH}), 1685$ (CO) cm ${ }^{-1}$; ${ }^{1} \mathrm{H}-\mathrm{NMR}$ (DMSO- $d_{6}$ ) $\delta 1.29\left(\mathrm{t}, J=7 \mathrm{~Hz}, 3 \mathrm{H}, \mathrm{CH}_{3}\right), 2.35$ (s, 3H, $\left.\mathrm{CH}_{3}\right), 4.27$ (q, $\left.J=7 \mathrm{~Hz}, 2 \mathrm{H}, \mathrm{CH}_{2}\right), 7.0-7.95(\mathrm{~m}, 10 \mathrm{H}, \mathrm{Ar}-\mathrm{H})$, 10.20 (s, 1H, NH, $\mathrm{D}_{2} \mathrm{O}$-exchangeable), 10.65 (s, 1H, NH, $\mathrm{D}_{2} \mathrm{O}$-exchangeable); MS m/z (\%) $372\left(\mathrm{M}^{+}\right.$, 47), 371 (20), 365 (60), 340 (60), 203 (13), 166 (100), 150 (40), 149 (80), 123 (80), 93 (100), 77 (73). Anal. Calcd. for $\mathrm{C}_{22} \mathrm{H}_{20} \mathrm{~N}_{4} \mathrm{O}_{2}$ (372.42): C, 70.95; H, 5.41; N, 15.04. Found: C, 70.72; H, 5.19; N, 14.84\%.

2-(4-Methylphenylhydrazono)-1,4-dihydro-naphtho[1,8-ef][1,4]diazepine-3-one (5a). Orange solid $(80 \%) ; \mathrm{mp} 100{ }^{\circ} \mathrm{C}(\mathrm{EtOH})$; IR (KBr) $v_{\max } 3325,3215,3120(3 \mathrm{NH}), 1658(\mathrm{C}=\mathrm{O}) \mathrm{cm}^{-1}$; ${ }^{1} \mathrm{H}-\mathrm{NMR}$ $\left(\mathrm{DMSO}-d_{6}\right) \delta 2.30\left(\mathrm{~s}, 3 \mathrm{H}, \mathrm{CH}_{3}\right), 7.05-7.67$ (m, 10H, Ar-H), 9.95 (s, 1H, NH, D 2 O-exchangeable), 10.15 (s, 1H, NH, $\mathrm{D}_{2} \mathrm{O}$-exchangeable), 10.91 (s, 1H, NH, $\mathrm{D}_{2} \mathrm{O}$-exchangeable); MS m/z (\%) $316\left(\mathrm{M}^{+}\right.$, 5), 315 (2), 225 (27), 197 (13), 134 (13), 106 (100), 105 (23), 91 (46), 89 (15), 77 (25). Anal. Calcd. for $\mathrm{C}_{19} \mathrm{H}_{16} \mathrm{~N}_{4} \mathrm{O}$ (316.36): C, 72.13; H, 5.10; N, 17.71. Found: C, 71.96; H, 4.87; N, 17.50\%.

2-(4-Chlorophenylhydrazono)-1,4-dihydro-naphtho[1,8-ef][1,4]diazepine-3-one (5b). Yellow solid (83\%); mp 120-122 ${ }^{\circ} \mathrm{C}(\mathrm{EtOH})$; IR (KBr) $v_{\max } 3315,3204,3210(3 \mathrm{NH}), 1660(\mathrm{C}=\mathrm{O}) \mathrm{cm}^{-1}$; ${ }^{1} \mathrm{H}-\mathrm{NMR}$ $\left(\mathrm{DMSO}-d_{6}\right) \delta$ 6.94-7.85 (m, 10H, Ar-Hs), 9.87 (s, 1H, NH, $\mathrm{D}_{2} \mathrm{O}$-exchangeable), 10.21 (s, 1H, NH, $\mathrm{D}_{2} \mathrm{O}$ exchangeable), 10.57 (s, $1 \mathrm{H}, \mathrm{NH}, \mathrm{D}_{2} \mathrm{O}$ exchangeable); $\mathrm{MS} m / z(\%) 338\left(\mathrm{M}^{+}+2,10\right), 337\left(\mathrm{M}^{+}+1,1\right)$, $336\left(\mathrm{M}^{+}, 35\right), 168$ (100), 140 (25), 114 (11), 111 (1), 84 (19). Anal. Calcd. For $\mathrm{C}_{18} \mathrm{H}_{13} \mathrm{ClN}_{4} \mathrm{O}$ (336.77): C, 64.19; H, 3.89; N, 16.64. Found: C, 64.35; H, 3.66; N, 16.48\%. 
Synthesis of 2-(2-hydroxybenzoylmethylene)-1,2-dihydro-perimidine (8). A mixture of 1,8-diaminonaphthalene (1) $(1.58,10 \mathrm{mmol})$ and 4-hydroxycoumarin $(6,1.62 \mathrm{~g}, 10 \mathrm{mmol})$ in ethanol $(20 \mathrm{~mL})$ was heated to reflux for $5 \mathrm{~h}$, then cooled. The solid formed was collected by filtration and washed with ethanol, then crystallized from a dioxane/ethanol mixture to give compound $\mathbf{8}$ as golden yellow crystals, $(89 \%), \mathrm{mp} 300{ }^{\circ} \mathrm{C}$, IR (KBr) $v_{\max }$ 3490, 3220, $3116(2 \mathrm{NH}, \mathrm{OH}), 1647$ (CO) cm ${ }^{-1}$; ${ }^{1} \mathrm{H}-\mathrm{NMR}$ (DMSO-d $d_{6} 5.62(\mathrm{~s}, 1 \mathrm{H}, \mathrm{CH}), 6.63-7.572(\mathrm{~m}, 10 \mathrm{H}, \mathrm{Ar}-\mathrm{H}), 10.91(\mathrm{~s}, 1 \mathrm{H}, \mathrm{NH}), 12.63(\mathrm{~s}, 1 \mathrm{H}, \mathrm{OH})$, $13.78(\mathrm{~s}, 1 \mathrm{H}, \mathrm{NH}) ;{ }^{13} \mathrm{C}-\mathrm{NMR}$ (DMSO- $\left.d_{6}\right): 77.15(=\mathrm{CH}), 106.0,116.37,117.76,118.41,119.11$, 119.52, 120.26, 126.81, 128.34, 132.99, 134.13, 153.50, 161.22 (Ar-C), $186.22(\mathrm{C}=\mathrm{O}) . \mathrm{MS} \mathrm{m} / z(\%)$ $302\left(\mathrm{M}^{+}, 67\right), 301$ (47), 182 (100), 168 (20), 127 (19), 121 (45), 114 (12), 93 (12). Anal. Calcd. For $\mathrm{C}_{19} \mathrm{H}_{14} \mathrm{~N}_{2} \mathrm{O}_{2}$ (302.33) C, 75.48; H, 4.67; N, 9.27. Found: C, 75.25; H, 4.41; N, 9.46\%.

Reaction of compound 8 with hydrazonoyl chlorides $4 \mathbf{a}-\mathbf{h}$. To a mixture of compound 8 (5 mmol) and the appropriate hydrazonoyl chloride $\mathbf{4 a}-\mathbf{h}(5 \mathrm{mmol}$ of each) in dioxane $(30 \mathrm{~mL})$ was added triethylamine $(0.7 \mathrm{~mL})$ and the mixture was heated to reflux for $10 \mathrm{~h}$, then cooled. The solid produced was collected by filtration and crystallized from the appropriate solvent to give the corresponding compounds 11a-h.

8-(2-Hydroxybenzoyl)-7H-9-(4-methylphenylhydrazono)-pyrrolo[1,2-a] perimidin-10-one (11a). Orange solid, $(82 \%) ; \mathrm{mp}>320{ }^{\circ} \mathrm{C}$ (ethanol/dioxane), IR (KBr) $v_{\max } 3200-3100$ (2NH, OH), 1690, 1639 $(2 \mathrm{C}=\mathrm{O}) \mathrm{cm}^{-1},{ }^{1} \mathrm{H}-\mathrm{NMR}\left(\mathrm{DMSO}-d_{6}\right) \delta: 2.19\left(\mathrm{~s}, 3 \mathrm{H}, \mathrm{CH}_{3}\right), 6.73-7.67$ (m, 14H, Ar-H), 9.89 (s, 1H, NH), 12.04 (s, 1H, OH), 13.80 (s, 1H, NH); MS m/z (\%) $460\left(\mathrm{M}^{+}, 13\right), 459$ (11), 458 (18), 340 (17), 339 (44), 338 (52), 283 (9), 248 (7), 221 (6), 167 (15), 166 (27), 152 (21), 127 (26), 121 (43), 106 (40), 91 (54), 84 (98), 84 (64), 77 (37), 55 (100). Anal. Calcd. for $\mathrm{C}_{28} \mathrm{H}_{20} \mathrm{~N}_{4} \mathrm{O}_{3}$ (460.48): C, 73.03; H, 4.38; N, 12.17. Found: C, 73.31; H, 4.17; N, 11.97\%.

8-(2-Hydroxybenzoyl)-7H-9-(3-methylphenylhydrazono)-pyrrolo[1,2-a] perimidin-10-one (11b). Orange solid, (79\%); mp 288-290 ${ }^{\circ} \mathrm{C}$ (ethanol/dioxane), IR (KBr) $v_{\max } 3236,3100(2 \mathrm{NH}, \mathrm{OH}), 1689,1647$ $(2 \mathrm{C}=\mathrm{O}) \mathrm{cm}^{-1},{ }^{1} \mathrm{H}-\mathrm{NMR}\left(\mathrm{DMSO}-d_{6}\right) \delta: 2.20\left(\mathrm{~s}, 3 \mathrm{H}, \mathrm{CH}_{3}\right), 6.61-8.50(\mathrm{~m}, 14 \mathrm{H}, \mathrm{Ar}-\mathrm{H}), 9.85(\mathrm{~s}, 1 \mathrm{H}, \mathrm{NH})$, 11.70 (s, 1H, OH), 12.03 (s, 1H, NH); MS m/z (\%) $460\left(\mathrm{M}^{+}, 13\right), 459$ (15), 458 (15), 340 (15), 339 (38), 338 (100), 337 (35), 254 (24), 178 (21), 169 (29), 138 (29), 127 (29), 124 (18), 93 (27), 92 (63), 91 (38), 84 (18), 83 (21), 77 (59), 76 (32). Anal. Calcd. for $\mathrm{C}_{28} \mathrm{H}_{20} \mathrm{~N}_{4} \mathrm{O}_{3}$ (460.48): C, 73.03; H, 4.38; N, 12.17. Found: C, 73.25; H, 4.09; N, 11.91\%.

8-(2-Hydroxybenzoyl)-7H-9-phenylhydrazono-pyrrolo[1,2-a]perimidin-10-one (11c). Yellow solid, (85\%); mp: $275-277{ }^{\circ} \mathrm{C}$ (ethanol/dioxane), IR (KBr) $v_{\max } 3429,3249(2 \mathrm{NH}, \mathrm{OH}), 1689,1643(2 \mathrm{C}=\mathrm{O}) \mathrm{cm}^{-1}$, ${ }^{1} \mathrm{H}-\mathrm{NMR}\left(\mathrm{DMSO}-d_{6}\right) \delta: 6.77-8.47(\mathrm{~m}, 15 \mathrm{H}, \mathrm{Ar}-\mathrm{H}), 10.0(\mathrm{~s}, 1 \mathrm{H}, \mathrm{NH}), 11.70(\mathrm{~s}, 1 \mathrm{H}, \mathrm{OH}), 12.02(\mathrm{~s}, 1 \mathrm{H}$, $\mathrm{NH})$; MS m/z (\%) $446\left(\mathrm{M}^{+}, 27\right), 445$ (23), 444 (23), 175(18), 140 (18), 120 (32), 95 (27), 90 (32), 82 (41), 77 (32), 64 (100). Anal. Calcd. for $\mathrm{C}_{27} \mathrm{H}_{18} \mathrm{~N}_{4} \mathrm{O}_{3}$ (446.46): C, 72.64; H, 4.06; N, 12.55. Found: C, $72.51 ; \mathrm{H}, 4.13 ; \mathrm{N}, 12.29 \%$.

8-(2-Hydroxybenzoyl)-7H-9-(4-chlorophenylhydrazono)-pyrrolo[1,2-a] perimidin-10-one (11d). Orange solid, (83\%); mp 308-310 ${ }^{\circ} \mathrm{C}$ (ethanol/dioxane), IR (KBr) $v_{\max } 3430-3247$ (br. 2NH, OH), 1688, $1644(2 \mathrm{C}=\mathrm{O}) \mathrm{cm}^{-1},{ }^{1} \mathrm{H}-\mathrm{NMR}$ (DMSO-d $)$ $\delta: 6.70-8.39(\mathrm{~m}, 14 \mathrm{H}, \mathrm{Ar}-\mathrm{H}), 9.80(\mathrm{~s}, 1 \mathrm{H}, \mathrm{NH}), 9.98$ 
(s, 1H, OH), 11.94 (s, 1H, NH); MS m/z (\%) $480\left(\mathrm{M}^{+}, 11\right), 479$ (15), 339 (46), 338 (100), 337 (21), 254 (14), 178 (29), 169 (16), 138 (29), 127 (29), 111 (43), 92 (63), 91 (24), 76 (31). Anal. Calcd. for $\mathrm{C}_{27} \mathrm{H}_{17} \mathrm{ClN}_{4} \mathrm{O}_{3}$ (480.90): C, 67.43; H, 3.56; N, 11.65. Found: C, 67.20; H, 3.32; N, 11.45\%.

8-(2-Hydroxybenzoyl)-7H-9-(4-bromophenylhydrazono)-pyrrolo[1,2-a] perimidin-10-one (11e). Dark red solid, (84\%); mp 278-280 ${ }^{\circ} \mathrm{C}$ (ethanol/dioxane), IR (KBr) $v_{\max } 3413,3200,3150$ (2NH, OH), 1689, $1647(2 \mathrm{C}=\mathrm{O}) \mathrm{cm}^{-1},{ }_{1}^{1} \mathrm{H}-\mathrm{NMR}$ (DMSO-d $) \delta: 6.70-8.44(\mathrm{~m}, 14 \mathrm{H}, \mathrm{Ar}-\mathrm{H}), 9.61(\mathrm{~s}, 1 \mathrm{H}, \mathrm{NH}), 9.97$ (s, 1H, OH), 11.94 (s, 1H, NH); MS m/z (\%) $526\left(\mathrm{M}^{+}+1,20\right), 525\left(\mathrm{M}^{+}, 20\right), 524$ (25), $523(41), 505$ (25), 340 (29), 339 (94), 338 (100), 254 (29), 219 (16), 179 (22), 173 (31), 166 (67), 155 (37), 138 (29), 121 (69), 104 (27), 93 (27), 92 (39), 91 (37), 77 (39), 76 (43), 75 (43). Anal. Calcd. for $\mathrm{C}_{27} \mathrm{H}_{17} \mathrm{BrN}_{4} \mathrm{O}_{3}$ (525.35): C, 61.73; H, 3.26; N, 10.66. Found: C, 61.54; H, 3.05; N, 10.47\%.

8-(2-Hydroxybenzoyl)-7H-9-(3-nitrophenylhydrazono)-pyrrolo[1,2-a]perimidin-10-one (11f). Orange solid, (76\%); $\mathrm{mp}>320{ }^{\circ} \mathrm{C}$ (ethanol/dioxane), IR (KBr) $v_{\max } 3433,3235(2 \mathrm{NH}, \mathrm{OH}), 1687,1647$ $(2 \mathrm{C}=\mathrm{O}) \mathrm{cm}^{-1},{ }^{1} \mathrm{H}-\mathrm{NMR}\left(\mathrm{DMSO}-d_{6}\right) \delta: 6.86-8.43(\mathrm{~m}, 14 \mathrm{H}, \mathrm{Ar}-\mathrm{H}), 10.05(\mathrm{~s}, 1 \mathrm{H}, \mathrm{NH}), 12.20(\mathrm{~s}, 1 \mathrm{H}$, $\mathrm{OH}), 12.44$ (s, 1H, NH); MS m/z (\%) $491\left(\mathrm{M}^{+}, 20\right), 490$ (15), 489 (29), 473 (29), 340 (21), 339 (65), 338 (100), 337 (46), 253 (25), 184 (15), 166 (32), 152 (26), 121 (44), 93 (22), 76 (47). Anal. Calcd. for $\mathrm{C}_{27} \mathrm{H}_{17} \mathrm{~N}_{5} \mathrm{O}_{5}$ (491.45): C, 65.99; H, 3.49; N, 14.25. Found: C, 65.75; H, 3.21; N, 14.08\%.

8-(2-Hydroxybenzoyl)-7H-9-(4-nitrophenylhydrazono)-pyrrolo[1,2-a]perimidin-10-one (11g). Red solid, (81\%); mp 310-313 ${ }^{\circ} \mathrm{C}$ (dioxane), IR (KBr) $v_{\max } 3400,3350,3220(2 \mathrm{NH}, \mathrm{OH}), 1693,1647$ $(2 \mathrm{C}=\mathrm{O}) \mathrm{cm}^{-1},{ }^{1} \mathrm{H}-\mathrm{NMR}\left(\mathrm{DMSO}-d_{6}\right) \delta:$ 6.90-7.92 (m, 14H, Ar-H), $9.79(\mathrm{~s}, 1 \mathrm{H}, \mathrm{NH}), 11.82(\mathrm{~s}, 1 \mathrm{H}$, $\mathrm{OH}), 12.17$ (s, 1H, NH); MS m/z (\%) $491\left(\mathrm{M}^{+}, 5\right), 490$ (12), 489 (28), 473 (62), 338 (100), 253 (22), 179 (11), 166 (31), 152 (19), 121 (29), 104 (18), 93 (22), 76 (57). Anal. Calcd. for $\mathrm{C}_{27} \mathrm{H}_{17} \mathrm{~N}_{5} \mathrm{O}_{5}$ (491.45): C, 65.99; H, 3.49; N, 14.25. Found: C, 65.69; H, 3.30; N, 14.45\%.

8-(2-Hydroxybenzoyl)-7H-9-(4-acetylphenylhydrazono)-pyrrolo[1,2-a]perimidin-10-one (11h). Orange solid, (82\%); mp 298-300 ${ }^{\circ} \mathrm{C}$ (dioxane), IR (KBr) $v_{\max } 3350,3240,3100(2 \mathrm{NH}, \mathrm{OH}), 1700,1689$, $1647(3 \mathrm{C}=\mathrm{O}) \mathrm{cm}^{-1},{ }^{1} \mathrm{H}-\mathrm{NMR}\left(\mathrm{DMSO}-d_{6}\right) \delta: 2.47\left(\mathrm{~s}, 3 \mathrm{H}, \mathrm{CH}_{3}\right), 6.85-8.49(\mathrm{~m}, 14 \mathrm{H}, \mathrm{Ar}-\mathrm{H}), 9.84(\mathrm{~s}, 1 \mathrm{H}$, $\mathrm{NH}), 11.80$ (s, 1H, OH), 12.13 (s, 1H, NH); MS m/z (\%) $488\left(\mathrm{M}^{+}, 46\right), 338$ (55), 326 (55), 153 (46), 137 (64), 135 (73), 121 (82), 104 (64), 92 (55), 84 (64), 77 (91), 76 (73), 60 (100). Anal. Calcd. for $\mathrm{C}_{29} \mathrm{H}_{20} \mathrm{~N}_{4} \mathrm{O}_{4}$ (488.49): C, 71.30; H, 4.13; N, 11.47. Found: C, 71.14; H, 4.0; N, 11.19\%.

\subsection{ORAC Assay}

Scavenging activity was measured by using an ORAC assay [42]. Dilutions of the samples $\mathbf{3 c}-\mathbf{f}, \mathbf{h}-\mathbf{j}$, 8, and 11a-h and Trolox (as antioxidant reference compound) were incubated for 10 min at $37{ }^{\circ} \mathrm{C}$ in fluorescein (70 $\mathrm{nM}$ final concentration). After this incubation period, 2,2"-azobis(2-amidinopropane) dihydrochloride (12 $\mathrm{mM}$ final concentration) was added to the mixture. Fluorescence was read every $56 \mathrm{~s}$ for 98 min using a FLUOstar Optima (BMG Labtech, Headquarters, Germany) fluorometer. Area under curve values were calculated for each sample and compared with the AUC corresponding to Trolox. Results are expressed as $\mu \mathrm{M}$ of Trolox equivalents/mg of sample. 


\subsection{Human Cell Lines for Cytotoxicity Assays}

The human renal denocarcinoma (TK-10), the human breast adenocarcinoma (MCF-7) and the human melanoma (UACC-62) Cell lines were used in these experiments. The human tumour cytotoxicities were determined following protocols established by the NCI [43]. TK-10, MCF-7 and UACC-62 cell lines were cultured in RPMI 1640 medium (Bio Whittaker, Basel, Switzerland) containing $20 \%$ foetal calf serum (FCS), $2 \mathrm{mM}$ L-glutamine, $100 \mu \mathrm{g} / \mathrm{mL}$ penicillin and $100 \mu \mathrm{g} / \mathrm{mL}$ streptomycin. All cell lines were maintained at $37{ }^{\circ} \mathrm{C}$ in a $5 \% \mathrm{CO}_{2}$ atmosphere with $95 \%$ humidity. Maintenance cultures were passaged weekly, and the culture medium was changed twice a week.

\subsection{Testing Procedure and Data Processing}

The sulforhodamine B (SRB) assay is used for cell density determination, based on the measurement of cellular protein content. The method described here has been optimized for the toxicity screening of compounds to adherent cells in a 96-well format. Viable cells were counted using a Coulter counter and diluted with medium to give final densities of $15 \times 10^{4}, 5 \times 10^{4}$ and $100 \times 10^{4}$ cells $/ \mathrm{mL}$ for TK-10, MCF-7and UACC-62, respectively. After $24 \mathrm{~h}$, the cells were treated with the serial concentrations of extracts. One hundred microlitres per well of each concentration was added to the plates to obtain final concentration of $10^{-4}, 10^{-5}, 10^{-6}, 10^{-7}$ and $10^{-8} \mathrm{M}$. The final volume in each well was $200 \mu \mathrm{g} / \mathrm{mL}$. The plates were incubated for $48 \mathrm{~h}$.

\subsection{Sulphorhodamine B Method}

After an incubation period for $48 \mathrm{~h}$, cell monolayers are fixed with $50 \mu \mathrm{L}$ of cold $50 \%$ (wt/vol) trichloroacetic acid (TAC) and stained for $60 \mathrm{~min}$ at $4{ }^{\circ} \mathrm{C}$, after which the excess dye (SRB solution $0.4 \% \mathrm{w} / \mathrm{v}$ in $1 \%$ acetic acid) is removed after $30 \mathrm{~min}$ incubated, by washing repeatedly with $1 \%$ (vol/vol) acetic acid. The protein-bound dye is dissolved in $10 \mathrm{mM}$ Tris base solution for OD determination at $492 \mathrm{~nm}$ using a microplate reader. At the end, $\mathrm{GI}_{50}$ values (concentrations required to inhibit cell growth by 50\%), TGI (concentration resulting in total growth inhibition) and $\mathrm{LC}_{50}$ (concentration causing 50\% of net cell killing) were calculated according to the previously described protocols [43]. Two or three experiments were carried out for each extract or compound. The data are given as the mean of three different assays $\pm \mathrm{SE}$.

\section{Conclusions}

Highly efficient and simple methods were described for the preparation of two series of naphtho[1,8-ef][1,4]diazepines and pyrrolo[1,2-a]perimidines from 1,8-diaminonaphthalene and hydrazonoyl chlorides. The mechanism of formation of such products was discussed. The newly synthesized compounds were examined for in vitro activity against several human cancer cell lines, including TK-10, MCF-7 and UACC-62. Compound 11c was found to have the best inhibitory activity towards all the three cell lines, followed by $3 \mathbf{i}$ that caused inhibition of $50 \%$ of cell growth on all the cancer cell lines. 


\section{Acknowledgments}

The authors thank Emilia carretero Acame, Departament of Pharmacology, Faculty of Pharmacy, Complutense University of Madrid, Spain for their support.

\section{Conflicts of Interest}

The authors declare no conflict of interest.

\section{References}

1. Pandya, A.B.; Prajapati, D.G.; Pandya, S.S. Synthesis of novel Naphthalene COX inhibitors for anti-inflammatory activity. J. Appl. Pharm. Sci. 2012, 2, 226-232.

2. Mkpenie, V.; Ebong, G.; Obot, I.B.; Abasiekong, B. Evaluation of the effect of azo group on the biological activity of 1-(4-methylphenylazo)-2-naphthol. E-J. Chem. 2008, 5, 431-434.

3. Strom, M.B.; Haug, B.E.; Rekdal, O.; Skar, M.L.; Stensen, W.; Svendsen, J.S. Important structural features of 15-residue lactoferricin derivatives and methods for improvement of antimicrobial activity. Biochem. Cell Biol. 2002, 80, 65-74.

4. Antonini, I.; Polucci, P.; Magnano, A.; Sparapani, S.; Martelli, S. Rational design, Synthesis, and biological evaluation of bis(pyrimido[5,6,1-de]acridines) and bis(pyrazolo[3,4,5-k1]acridine-5carboxamides) as new anticancer agents. J. Med. Chem. 2004, 47, 5244-5250.

5. Akita, M.; Seto, H.; Aoyama, R.; Kimura, J.; Kobayashi, K. Novel rearrangements in the reactions directed toward preparation of spiro- $N, N$-ketals: Reactions of naphthalene-1,8-diamine with ninhydrin and isatin. Molecules 2012, 17, 13879-13890.

6. El-Shaieb, K.M. Condensation of 1-(dicyanomethylene)acenaphthene-2-one with aromatic diamines. J. Chin. Chem. Soc. 2008, 55, 1150-1155.

7. Cheeseman, G.W.H.; Eccleshall, S.A. Synthesis of pyrrolo[1,2-a]naphtho[1,8-ef][1,4]diazepines. J. Heterocycl. Chem. 1986, 23, 65-67.

8. Üngören, S.H. Transformation of furan-2,3-diones with 1,8-diaminonaphthalene to naphtho-[1,8ef][1,4]diazepin-2(1H)-ones. Synth. Comm. 2009, 39, 3657-3662.

9. Prakash, G.K.S.; Paknia, F.; Narayan, A.; Mathew, T.; Olah, G.A. Synthesis of perimidine and 1,5-benzodiazepine derivatives using tamed Brønsted acid, BF3-H2O. J. Fluor. Chem. 2013, 152, 99-105.

10. Luthin, D.R.; Rabinovich, A.K.; Bhumralkar, D.R.; Youngblood, K.L.; Bychowski, R.A.; Dhanoa, D.S.; May, J.M. Synthesis and biological activity of oxo-7H-benzo[e]perimidine-4carboxylic acid derivatives as potent, nonpeptide corticotropin releasing factor (CRF) receptor antagonists. Bioorg. Med. Chem. Lett. 1999, 9, 765-770.

11. Pozharskii, A.F.; Dalnikovskaya, V.V. Perimidines. Russ. Chem. Rev. 1981, 50, 816-835.

12. Claramunt, R.M.; Dotor, J.; Elguero, J. Structure, Reactivity and synthesis of perimidines derivatives (Dihydroperimidines and perimidinones). Ann. Quim. 1995, 91, 151-183.

13. Herbert, J.M.; Woodgate, P.D.; Denny, W.A. Potential antitumor agents. 53. Synthesis, DNA binding properties, and biological activity of perimidines designed as minimal DNA-intercalating agents. J. Med. Chem. 1987, 30, 2081-2086. 
14. Brana, M.F.; Garrido, M.; Rodriguez, M.; Morcillo, M.J.; Alvarez, Y.; Valladares, Y.; Klebe, G. Synthesis, Structure and cytostatic activity of a series of 2-substituted perimidines. Eur. J. Med. Chem. 1990, 25, 209-215.

15. Stefańska, B.; Dzieduszycka, M.; Bontemps-Gracz, M.M.; Borowski, E.; Martelli, S.; Supino, R.; Pratesi, G.; de Cesare, M.A.; Zunino, F.; Kuśnierczyk, H.; et al. 8,11-Dihydroxy-6[(aminoalkyl)amino]-7H-benzo[e]perimidin-7-ones with activity in multidrug-resistant cell lines: Synthesis and antitumor evaluation. J. Med. Chem. 1999, 42, 3494-3501.

16. Bu, X.; Deady, L.W.; Finlay, G.J.; Baguley, B.C.; Denny, W.A. Synthesis and cytotoxic activity of 7-Oxo-7 H-dibenz[f,ij] isoquinoline and 7-Oxo-7H-benzo[e]perimidine derivatives. J. Med. Chem. 2001, 44, 2004-2014.

17. Farghaly, T.A.; Mahmoud, H.K. Synthesis of pyrido[2,3- $d][1,2,4]$ triazolo[4,3- $a]$ pyrimidin-5-ones 3 as potential antimicrobial agents. Arch. Pharm. Chem. Life Sci. 2013, 346, 392-402.

18. Kima, S.-H.; Kimb, J.-H.; Cuia, J.-Z.; Galc, Y.-S.; Jind, S.-H.; Koh, K. Absorption spectra, aggregation and photofading behaviour of near-infrared absorbing squarylium dyes containing perimidine moiety. Dyes Pigments 2002, 55, 1-7.

19. Wan, X.; Lv, X.; He, G.; Yu, A.; Chen, Y. Synthesis of neutral stable polyradicals and their application on photovoltaic devices. Eur. Polym. J. 2011, 47, 1018-1030.

20. Bazinet, P.; Yap, G.P.A.; Richeson, D.S. Constructing a stable carbene with a novel topology and electronic framework. J. Am. Chem. Soc. 2003, 125, 13314-13315.

21. Herbst, W.; Hunger, K. Industrial Organic Pigments, 3rd ed.; Wiley-VCH: Weinheim, Germany, 2004; p. 9.

22. Nagao, Y.; Tsuda, K.; Kozawa, K.; Uchida, T. Synthesis and properties of benzimidazole and naphthoimidazole derivatives of perylenedicarboximide. Heterocycles 2001, 54, 757-764.

23. Alfredo, N.V.; Likhatchev, D.; Ramirez, S.B.; Vazquez, J.R.; Valverde, G.C.; Alexandrova, L. Highly effective low temperature route to pyrroloperimidines synthesis and their copolymerization with styrene and methyl methacrylate. Polymer 2008, 49, 3654-3662.

24. Mizuguchi, J. Crystal structure and electronic characterization of trans and cis perinone pigments. J. Phys. Chem. B 2004, 108, 8926-8930.

25. Abdel Hafez, N.A.; Farghaly, T.A.; Al-Omar, M.A.; Abdalla, M.M. Synthesis of bioactive polyheterocyclic ring systems as 5 $\alpha$-reductase inhibitors. Eur. J. Med. Chem. 2010, 45, 4838-4844.

26. Farghaly, T.A.; Gomha, S.M.; Abbas, E.M.; Abdalla, M.M. Hydrazonoyl halides as precursors for new fused heterocycles of $5 \alpha$-reductase inhibitors. Arch. Pharm. 2012, 345, 117-122.

27. Abdel-Aziz, H.A.; El-Zahabi, H.S.A.; Dawood, K.M. Microwave-assisted synthesis and in-vitro anti-tumor activity of 1,3,4-triaryl-5- $N$-arylpyrazole-carboxamides. Eur. J. Med. Chem. 2010, 45, 2427-2432.

28. Abdel-Aziz, H.A.; Mekawey, A.A.I.; Dawood, K.M. Convenient synthesis and antimicrobial evaluation of some novel 2-substituted-3-methylbenzofuran derivatives. Eur. J. Med. Chem. 2009, $44,3637-3644$.

29. Dawood, K.M.; Abdel-Gawad, H.; Ellithey, M.; Mohamed, H.A.; Hegazi, B. Synthesis, Anticonvulsant, and anti-inflammatory activities of some new benzofuran-based heterocycles. Arch. Pharm. Chem. Life Sci. 2006, 339, 133-140. 
30. Dawood, K.M.; Abdel-Gawad, H.; Ragab, E.A.; Ellithey, M.; Mohamed, H.A.; Synthesis, Anticonvulsant, and anti-inflammatory evaluation of some new benzotriazole and benzofuran-based heterocycles. Bioorg. Med. Chem. 2006, 14, 3672-3680.

31. Farghaly, T.A.; Abdallah, M.A.; Aziz, A.M.R. Synthesis and antimicrobial activity of some new 1,3,4-thiadiazole derivatives. Molecules 2012, 17, 14625-14636.

32. Riyadh, S.M.; Farghaly, T.A.; Abdallah, M.A.; Abdalla, M.M.; Abd El-Aziz, M.R. New pyrazoles incorporating pyrazolylpyrazole moiety: Synthesis, Anti-HCV and antitumor activity. Eur. J. Med. Chem. 2010, 45, 1042-1050.

33. Farghaly, T.A.; Abdalla, M.M. Synthesis, Tautomerism, Antimicrobial, Anti-HCV, Anti-SSPE, Antioxidant and antitumor activities of arylazobenzosuberones. Bioorg. Med. Chem. 2009, 17, 8012-8019.

34. Karc1, F.; Sener, N.; Yamac, M.; Sener, I.; Demircal1, A. The synthesis, antimicrobial activity and absorption characteristics of some novel heterocyclic disazo dyes. Dyes Pigments 2009, 80, 47-52.

35. Hamdi, M.; Grech, O.; Sakellariou, R.; Speziale, V. New method of synthesis of 1,5-benzodiazepin-2-ones from 4-hydroxycoumarin. J. Heterocycl. Chem. 1994, 31, 509-511.

36. Hamdi, N.; Lidrissi, C.; Saoud, M.; Nievas, R.A.; Zarrouk, H. Synthesis of some new biologically active coumarin derivatives. Chem. Heterocycl. Compd. 2006, 42, 320-325.

37. Üngören, S.H.; Koca, T.; Yilmaz, F. Preparation of perinones via a novel multicomponent synthesis of isoindole scaffold. Tetrahedron 2011, 67, 5409-5414.

38. Paragamian, V.; Baker, M.B.; Puma, B.M.; Reale, J. A study of the synthesis and some reactions of perimidines. J. Heterocycl. Chem. 1968, 5, 591-597.

39. Cado, F.; Di-Martino, J.; Jacquault, P.; Bazureau, J.P.; Hamelin, J. Amidine-enediamine tautomerism: Addition of isocyanates to 2-substituted $1 H$-perimidines. Some syntheses under microwave irradiation. Bull. Soc. Chim. Fr. 1996, 133, 587-595.

40. Shawali, A.S.; Zayed, M.M.; Farghaly, T.A. Synthesis and biological activity of new $1 H$-pyrazolo[3,4-b]quinoxalines (Flavazoles). J. Heterocycl. Chem. 2005, 42, 185-189.

41. Battin, E.E.; Brumaghim, J.L. Antioxidant activity of sulfur and selenium: A Review of reactive oxygen species scavenging, glutathione peroxidase, and metal binding antioxidant mechanisms. Cell Biochem. Biophys. 2009, 55, 1-23.

42. Davalos, A.; Gomez-Cordoves, C.; Bartolome, B. Extending applicability of the oxygen radical absorbance capacity (ORAC-fluorescein) assay. J. Agric. Food Chem. 2004, 52, 48-54.

43. Monks, A.; Scudeiro, D.; Skehan, P.; Shoemaker, R.; Paull, K.; Vistica, D.; Hose, C.; Langley, J.; Cronise, P.; Vaigro-Wolff, A.; et al. Feasibility of a high-flux anticancer drug screen using a diverse panel of cultured human tumor cell lines. J. Natl. Cancer Inst. 1991, 83, 757-766.

Sample Availability: Samples of the compounds 3, 5, 8 and $\mathbf{1 1}$ are available from the authors.

(C) 2014 by the authors; licensee MDPI, Basel, Switzerland. This article is an open access article distributed under the terms and conditions of the Creative Commons Attribution license (http://creativecommons.org/licenses/by/3.0/). 